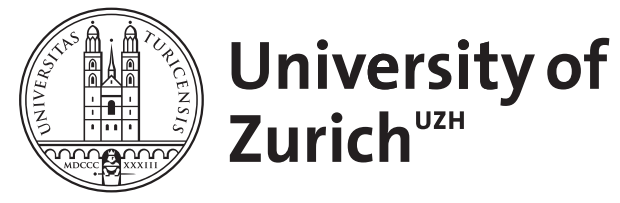

\title{
Excimer Laser Trabeculostomy (EL T): An Effective MIGS Procedure for Open-Angle Glaucoma
}

\author{
Berlin, M S ; Töteberg-Harms, M ; Kim, E ; Vuong, I ; Giers, U
}

Posted at the Zurich Open Repository and Archive, University of Zurich ZORA URL: https://doi.org/10.5167/uzh-98736

Book Section

Originally published at:

Berlin, M S; Töteberg-Harms, M; Kim, E; Vuong, I; Giers, U (2014). Excimer Laser Trabeculostomy (EL T): An Effective MIGS Procedure for Open-Angle Glaucoma. In: Samples, J R; Ahmed, I I K. Surgical Innovations in Glaucoma. New York: Springer Science + Business Media New York, 85-95. 


\title{
Excimer Laser Trabeculostomy (ELT): An Effective MIGS Procedure for Open-Angle Glaucoma
}

\author{
Michael S. Berlin, Marc Töteberg-Harms, Edward Kim, \\ Iris Vuong, and Ulrich Giers
}

Elevated intraocular pressure (IOP) in most open-angle glaucoma is due to an obstruction of aqueous outflow thought to be localized predominantly at the juxtacanalicular trabecular meshwork (TM) and the inner wall of Schlemm's canal (SC). There have been multiple surgical attempts to directly treat this pathology by creating channels to connect the anterior chamber to SC. Various iterations have included mechanical drilling techniques, thermal laser and alternative thermal cautery techniques, and a variety of tube implants with the common goal of bypassing this increased outflow resistance. However, the majority of these techniques concurrently or subsequently create sufficient adjacent tissue disruption to eventuate in inflammatory healing responses adequate to overcome the benefits of the procedures, resulting in midterm or long-term failures. To overcome these known causes of failure, technologies and techniques for the purpose of creating such channels were sought which did not cause scar tissue formation. The requirements include minimal tissue disruption during the process of creating the openings and channels which are of adequate size and number to enable outflow but not so large as to alter the aqueous composition.

\section{M.S. Berlin, MD, MS ( $\square)$ \\ Glaucoma Institute of Beverly Hills, Jules Stein \\ Eye Institute, UCLA, 8733 Beverly Blvd., \\ Suite 301, Los Angeles, CA 90048, USA \\ e-mail: berlin@ucla.edu}

M. Töteberg-Harms, MD

Department of Ophthalmology, University Hospital Zurich, Frauenklinikstrasse 24, Zurich 8091, Switzerland

e-mail: marctoeteberg@aol.com

\section{E. Kim, BA $\bullet$ I. Vuong}

Glaucoma Institute of Beverly Hills, 8733 Beverly Blvd., Suite 301, Los Angeles, CA 90048, USA

e-mail: ehkim36@gmail.com; irisvuong@gmail.com

\section{U. Giers, MD}

Augen-klinik OWL, Detmold, Elisabethstrasse 85,

32756 Detmold, Germany

e-mail: ugiers@doc4eye.de
The excimer laser trabeculostomy (ELT) concept utilizes a 308-nm xenon chloride excimer laser to provide a precise and essentially nonthermal approach to improve outflow in a manner which does not provoke a healing response. ELT is a procedure performed via a clear corneal incision, sparing conjunctiva, in which direct visualization of the target tissue via a goniolens or an endoscope provides immediate feedback to the surgeon. ELT, when compared to the more invasive glaucoma surgical procedures such as trabeculectomy, is almost as efficacious in both IOP-lowering and decreasing pressure-lowering medication use, while being far less traumatic. The sparing of conjunctiva is a major advantage of this technique, because success rates of a subsequent trabeculectomy, if ever necessary, would not be compromised. This microinvasive glaucoma surgery (MIGS) procedure, another option in the armamentarium for the glaucoma surgeon, has a high safety profile, rapid stabilization, and clinical verification of long-term efficacy with minimal impact on quality of life. It may also become a replacement option for topical glaucoma medicinal therapies eliminating their associated cost, compliance issues, and side effects from their long-term use.

\section{What Are MIGS Procedures and Where Do They Fit in Glaucoma Treatment Paradigms?}

The invasive procedure and lifestyle altering sequelae of trabeculectomy limit the use of this procedure to later stage and recalcitrant glaucoma patients. Issues regarding medication costs, compliance, and toxicity of preservatives suggest medicinal therapeutic options also have limitations. Thus, there remains an unmet patient need for treatments that could effectively treat mild to moderate glaucoma. In recent years, several MIGS procedures have been developed to address these limitations of traditional treatment options. All have advantages and disadvantages, but no current technique fulfills all requirements which are (1) ab interno microincision, (2) minimal trauma, (3) efficacy, (4) high safety profile, and (5) rapid recovery [1]. 
1. MIGS procedures use an ab interno micro-incisional approach through a clear corneal incision of less than $2 \mathrm{~mm}$, which spares the conjunctiva and thus prevents significant external scarring, allowing future unaffected conjunctival surgery, if needed. This also allows for direct visualization of anatomic landmarks to optimize placement of a device, incision or excision within the angle and is easily combined with cataract surgery. Furthermore, a micro-incision facilitates the intra-operative maintenance of the anterior chamber, retention of normal ocular anatomy, minimizes changes in refractive outcome, and adds to procedural safety.

2. MIGS procedures are minimally traumatic to the target tissue, with negligible disruption of normal anatomy and physiology. Devices are biocompatible and ideally enhance physiologic outflow pathways.

3. MIGS procedures must be effective in both lowering IOP and reducing medication use.

4. MIGS procedures are characterized by an extremely high safety profile. Many MIGS procedures are less efficacious when compared to more invasive glaucoma surgeries such as trabeculectomy with antimetabolites or glaucoma drainage devices. However, this compromise in efficacy is balanced by their low-risk profile. MIGS procedures are associated with a decreased incidence of serious complications that are often associated with other glaucoma surgeries including hypotony, choroidal effusions, suprachoroidal hemorrhage, anterior chamber shallowing, corneal decompensation, cataract formation, diplopia, and bleb-related complications such as bleb dysesthesia and endophthalmitis.

5. MIGS procedures have a rapid recovery with minimal impact on the patient's quality of life.

In conclusion, MIGS procedures fit for mild do moderate glaucoma cases, in which an IOP in low to mid teens is sufficient. In addition, a reduction in IOP lowering medication is desired. The safety profile of the procedure as well as a rapid recovery is important.

\section{Previous Meshwork Surgeries and Their Disadvantages}

Current MIGS procedures can successfully bypass the TM in order to create direct flow from the anterior chamber into SC unlike earlier attempts to address this anatomic pathology, which often failed. Procedures utilizing mechanical devices and lasers to perforate the TM have been shown to adequately bypass the outflow obstruction for the short term but have been unsuccessful in the long term due to the amount of adjacent tissue damage related to the nature of the technique or device. The adjacent tissue damage evokes a healing response which eventually closes the channels. As laser technology evolved, each new laser was applied for this purpose, but none enabled long-term patent openings. Krasnov et al. showed moderate success using a ruby laser $(943 \mathrm{~nm})$ to perform "trabeculopuncture" [2]. Other laser trabeculopuncture attempts including Hager's [3] use of an argon laser $(488+514 \mathrm{~nm})$ and Fankhauser's [4] use of a neodymium:yttrium-aluminum-garnet (Nd:YAG) laser (continuous wave $1,064 \mathrm{~nm}$ ) have also been unsuccessful due to scarring. These and other laser trabeculopuncture attempts also limit prospective options for subsequent procedures $[5,6]$ should they become necessary as they induce destruction of local tissue and scar formation of adjacent tissue, often involving large regional areas. In addition, with large openings and markedly increased outflow, the compositional alterations of the aqueous would supplement the tissue destructive healing responses.

Following these initial attempts using lasers ab interno to perforate TM or to create full-thickness sclerostomies, Wise et al. determined that a continuous wave, essentially long pulsed argon laser $(488+514 \mathrm{~nm})$ could successfully modify the TM to increase outflow without perforation. Their argon laser trabeculoplasty (ALT) procedure effectively lowered IOP, but it functioned by creating thermal damage to the target tissue, causing coagulative necrosis of the TM [7]. In contrast to ALT, laser trabeculoplasty (LTP) is now more commonly performed with solid-state (532, frequencydoubled Nd:YAG) and diode $(810 \mathrm{~nm})$ lasers. Studies comparing efficacy of these thermal lasers demonstrate minimal differences in efficacy, longevity, or repeatability.

The efficacy of LTP in lowering IOP has been well documented in the literature [8-10]. However, long-term studies have shown that the IOP-lowering efficacy of LTP decreases over time, from $77 \%$ success rate at 1 year, to $49 \%$ at 5 years, and finally, to $32 \%$ at 10 years [11]. Additionally, because of the significant TM scarring caused by ALT, repeat treatments are not recommended and have not proven successful clinically [12]. LTP causes thermal coagulative damage to the uveoscleral meshwork, disruption of the trabecular beams, and heat damage to the surrounding collagen fibers. This thermal damage is in part responsible for the inflammatory response, scarring of the TM tissue, peripheral anterior synechiae, and IOP spikes sometimes observed in eyes which have undergone LTP [13].

In contrast to the diffuse thermal effects of argon, solidstate, and diode laser with relatively long pulse durations in the range of $0.1 \mathrm{~s}$, recent advances in LTP utilize lasers with shorter pulse durations of 3-10 ns. Selective laser trabeculoplasty (SLT), based on the principle of selective photothermolysis, relies on selective absorption of short laser pulses to generate and spatially confine heat to pigmented targets within TM cells [14, 15]. SLT uses a Q-switched, frequencydoubled 532-nm Nd:YAG laser. Q-switching of the laser allows for an extremely brief and thus high-powered light pulse to be delivered to the target tissues. The short duration of the pulse is critical in preventing collateral damage to the 
surrounding tissues [16]. The mechanisms of the pressure effects following ALT, LTP, and SLT which alter but do not perforate TM are yet undetermined.

Preserving the TM may become more important in the near future, as surgical techniques are developed to operate directly on SC or the juxtacanalicular TM. Thermal LTP would preclude the use of the newer MIGS procedures in these patients, as their TM and adjacent tissues, including SC would be likely to have been damaged. Once methods of preoperative evaluations of the patency of the required outflow pathways are clinically useful, such patients can be better evaluated as candidates for MIGS procedures.

\section{The Concept of ELT}

Ultraviolet excimer laser photoablation enables the precise removal of targeted tissue with meticulous local and adjacent temperature control to prevent thermal damage to surrounding tissues, exemplified by the use of 193-nm excimer lasers for corneal surface ablation, which facilitates successful refractive surgery. However, the 193-nm wavelength, although precise and non-tissue damaging, is not readily transmissible via fiber optics and can therefore not be used for intracameral procedures. In contrast, 308-nm excimer laser generated light is amenable to fiber-optic transmission and, after extensive preclinical experimentation, became the wavelength of choice for non-thermal, precisely targeted $a b$ interno fistulizing procedures [17]. The applications of this non-thermal, ab interno, fiber-optic-delivered energy evolved, initially from full-thickness sclerostomy, creating a bleb via an ab interno approach, subsequently to trabeculostomy, once the parameters of the target tissue anatomy ${ }^{1}[18,19]$, localization of SC, and ablation rates for this wavelength in this tissue were determined and idealized to enable a successful procedure to bypass TM and juxtacanalicular TM obstructions allowing and improving physiological outflow directly into SC.

Initial in vitro experiments led to animal trials [20]. In a study of the effects of 308-nm excimer laser energy applied ab interno to the limbal sclera of rabbit eyes, long-term decreases in IOP were achievable. The use of this 308 -nm wavelength, unlike that of earlier trials with thermal lasers, enables lasertissue interactions with significant advantages. This excimer

\footnotetext{
${ }^{1}$ Target tissue anatomic considerations to minimize healing responses must specifically address minimizing trauma to the outer wall of SC. The outer wall endothelium contains fibroblasts, whereas the inner wall endothelium does not. Thus, avoiding trauma to the outer wall and thereby not stimulating a fibroblast response is paramount to the successful maintenance of outflow. Another anatomic consideration is the space between the inner and outer walls of SC, which can be less than $20 \mu \mathrm{m}$. The accuracy of the tool used to enter the inner wall such that it does not disturb the outer wall must be of this same scale. The ablation precision of $308 \mathrm{~nm}$ on this tissue, unlike that of lasers and devices utilized in earlier attempts to fistulize SC, facilitates the non-thermal and accurate tissue removal which thereby enables the ELT procedure's efficacy.
}

laser is less likely to evoke a cicatricle response in the TM or sclera than those seen in trials using visible or infrared lasers, which cause thermal tissue damage and subsequent healing responses. In addition, there is minimal exposure of adjacent tissue to radiation enabled by direct contact of the laser energy to the target tissue via the fiber-optic delivery system. With evidence that TM and scleral tissue could be successfully removed without adjacent tissue damage, without subsequent scar formation or channel closure, and with ablation accuracy which would enable precise targeting of TM, juxtacanalicular $\mathrm{TM}$, and the inner wall of SC without perforating the outer wall of SC, these studies formed the basis for the development of the current ELT technology and techniques [21].

\section{ELT Technique}

ELT, first used clinically in 1997 by Vogel and Lauritzen, treats the pathology responsible for most open-angle glaucoma by decreasing the outflow resistance at the juxtacanalicular TM and the inner wall of SC [22]. It is performed with a short-pulsed (80 ns) 308-nm xenon chloride (XeCl) excimer laser which delivers photoablative energy to precisely remove the tissue which obstructs aqueous outflow with minimal thermal damage to adjacent tissue [23]. ELT surgery is performed as an outpatient procedure, under topical, peribulbar, or retrobulbar local anesthesia. Following a paracentesis and stabilization of the anterior chamber with a viscoelastic agent, the surgeon introduces a fiber-optic probe, which is advanced across the anterior chamber to contact the TM (Fig. 8.1). Probe placement is controlled by direct observation using either a goniolens or an endoscope. Four to ten channels are created into SC (Fig. 8.2). The probe is then removed, the viscoelastic agent is replaced by balanced salt solution, and the patient is monitored postoperatively. Most commonly the probe insertion is superotemporal and the laser channels inferonasal. Variations include spacing the channels over both inferior quadrants, creating temporary hypotony following removal of the probe to enable enumeration of the number of patent channels into SC by observing an induced retrograde blood reflux followed by repressurization, among others. The blood reflux is a common but inconsequential occurrence (Fig. 8.3). To date no studies have shown benefit from treatment in more than one quadrant. As no single protocol has been established, each surgeon tends to create their own technique. In spite of these numerous variations, the outcomes are remarkably similar.

By means of the essentially non-thermal photoablation using the specified laser parameters, ELT evaporates human tissue, denaturing the organic structures without producing undesirable marginal necrosis [24]. ELT excises the TM, the juxtacanalicular TM, and the inner wall of SC without damaging the outer wall of SC or the collector channels [25]. No filtering fistula or bleb is created [21, 26, 27]. 

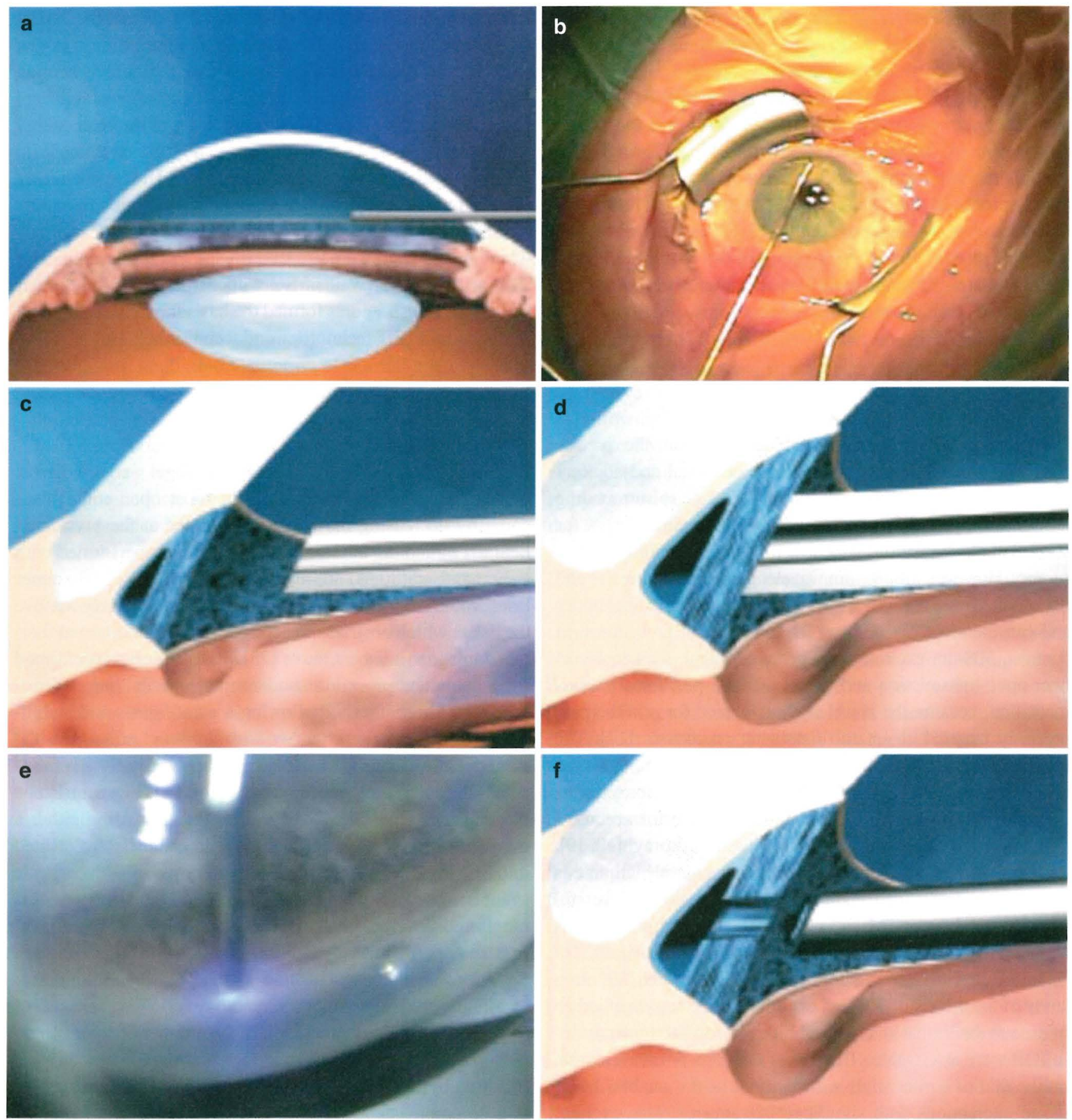

Fig. 8.1 Drawings and photos of the excimer laser trabeculostomy procedure. The laser fiber is introduced into the anterior chamber through a clear cornea incision (a) and advanced across the anterior chamber (b) to the trabecular meshwork of the opposite quadrant (c). When the fiber tip is in contact with or slightly compresasing the

trabecular meshwork laser pulses are initiated (d). Tissue fluorescence produced by each UV laser pulse can be visualized gonioscopicly (e). A series of laser channels of approx. $0.2 \mathrm{~mm}$ diameter each enabels flow from the anterior chamber to Schlemm's canal (f). a, c-d, and f: Peschke $\mathrm{GmbH}$, Waldshut-Tiengen, Germany; b and e: U. Giers

\section{Technical Aspects of Performing an ELT Procedure}

The current ELT procedure is performed with an AIDA XeCl laser (TUI-Laser AG, Germering, Germany) in the following manner:

1. The laser is automated to internally calibrate and control the output fluence in accordance with the manufacturer's specifications. Unlike solid-state lasers, this $\mathrm{XeCl}$ gas laser requires a short "warm up" time during which the output energy is stabilized before use. 


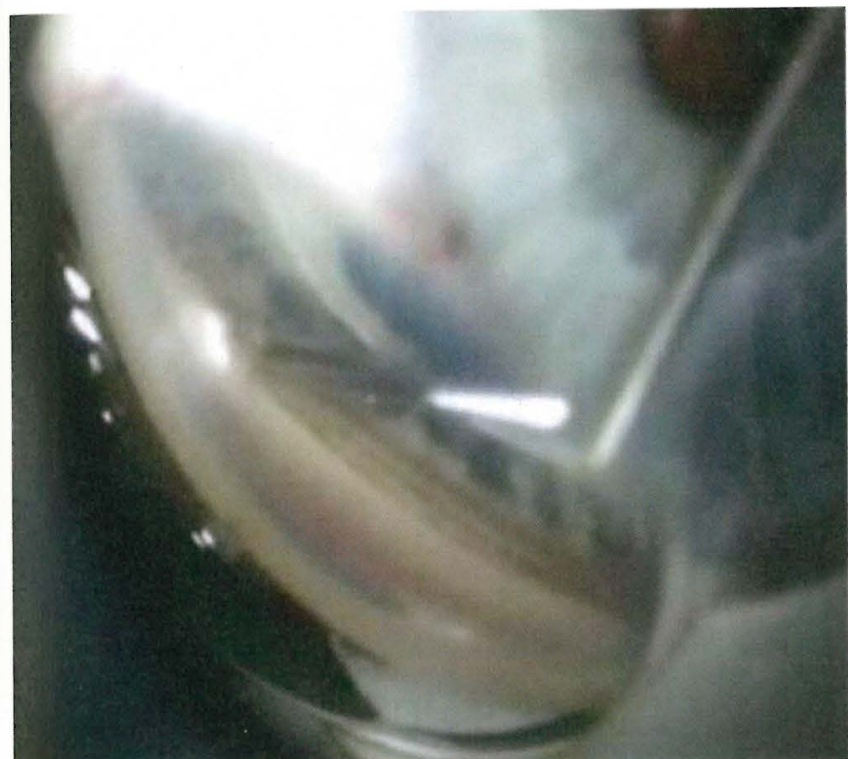

Fig. 8.2 Gonioscope view/IOP high $=\mathrm{SC}$ not visible (U. Giers)

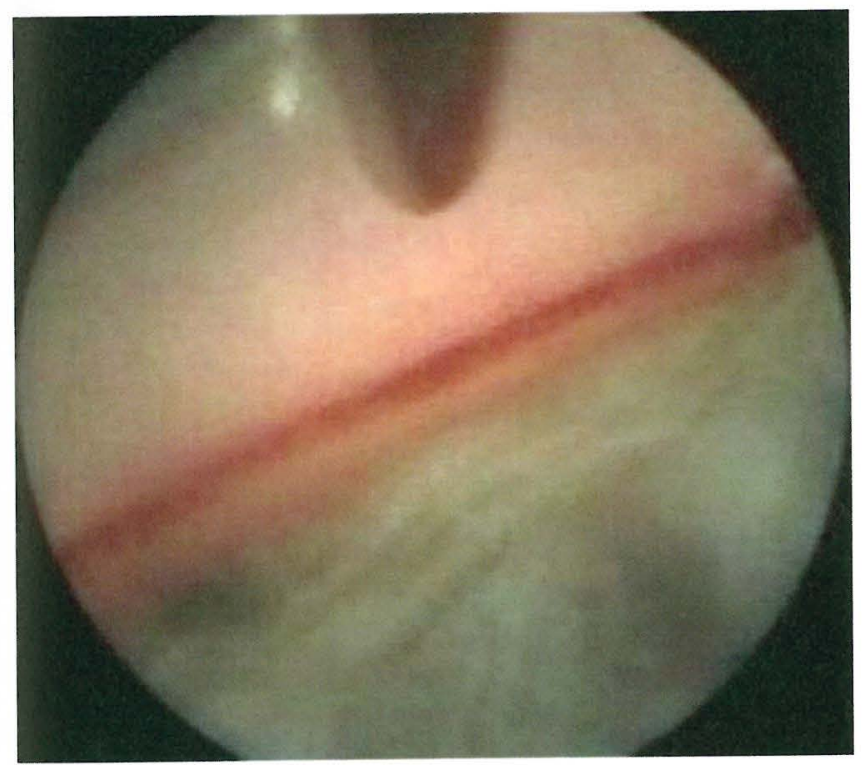

Fig. 8.3 Endoscopic view/IOP low =SC visible, blood filled (U. Giers)

2. The sterile, non-reusable fiber-optic delivery system is coupled to the laser. The output beam is then adjusted at the fiber tip to ensure suprathreshold fluence for tissue ablation at the fiber tip. The console includes a power meter to enable this calibration which is performed prior to each procedure, similar to the tuning of a phaco handpiece before use.

3. Topical, peribulbar, or retrobulbar local anesthesia is administered. In ELT alone, topical pilocarpine $2 \%$ is used preoperatively to constrict the pupil. In phakic patients this may also assist in protecting the lens. Alternatively, intracameral Miochol (acetylcholine $10 \mathrm{mg} / \mathrm{ml}$ ) may be used.

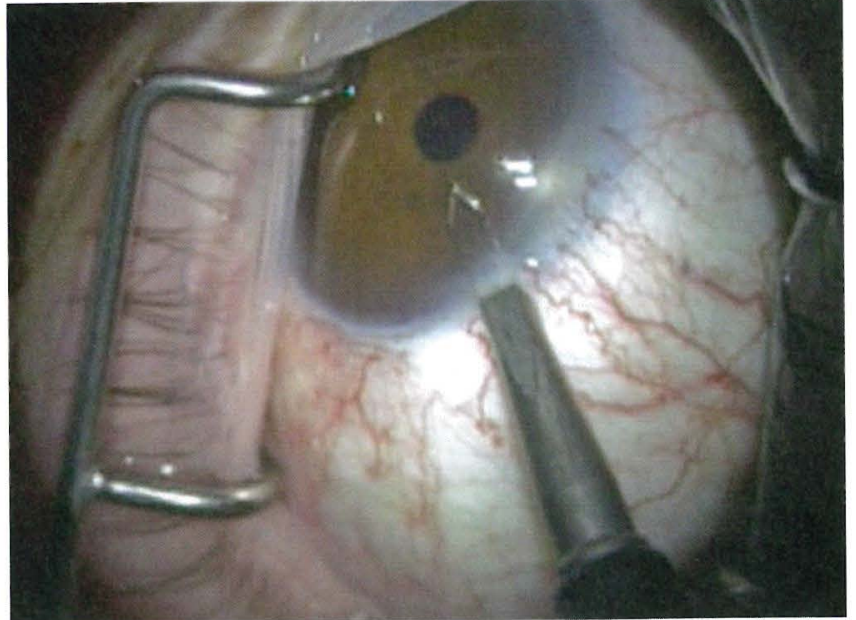

Fig. 8.4 Paracentesis in the superotemporal perilimbal cornea (U. Giers)

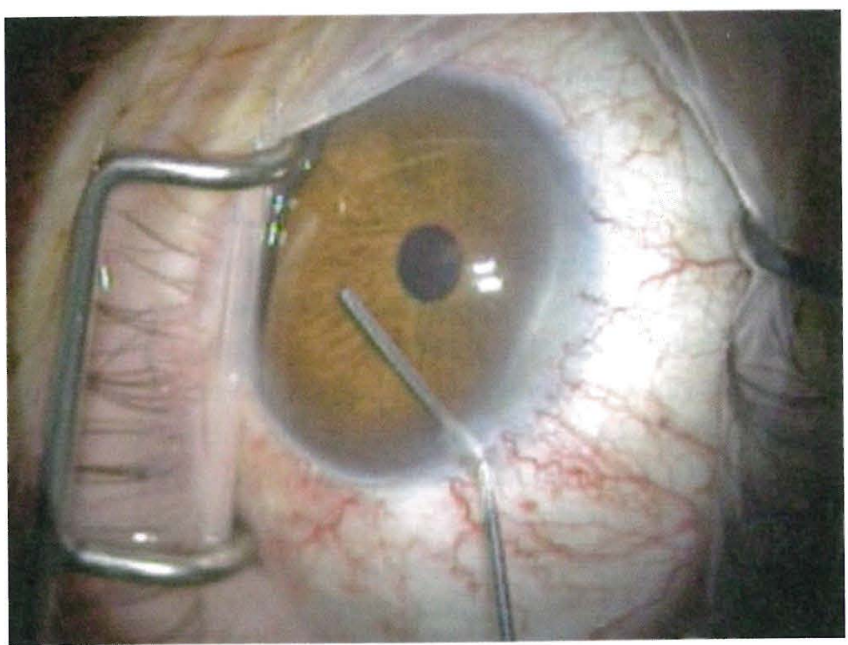

Fig. 8.5 Viscoelastic agent is introduced (U. Giers)

4. A $1 \mathrm{~mm}$ paracentesis is created in the superotemporal perilimbal cornea 2 o'clock for left eyes and 10 o'clock for right eyes, or in combined cataract and ELT procedures, the previously created phaco-tunnel may be used in a similar fashion (Fig. 8.4).

5. A viscoelastic agent (Healon) fills the anterior chamber through the paracentesis. Depending on the surgeons' preference, the IOP may be unchanged or increased by this injection, enabling or precluding visualization of SC by blood reflux (Fig. 8.5).

6. The laser probe is inserted into the anterior chamber through the paracentesis (Fig. 8.6) and is advanced to the opposite chamber angle via gonioscopic (using the surgeon's preferred goniolens) or endoscopic visualization (Fig. 8.7). The ELT fiber may be attached coaxial with an endoscope, or a second paracentesis endoscopic view may be utilized. 


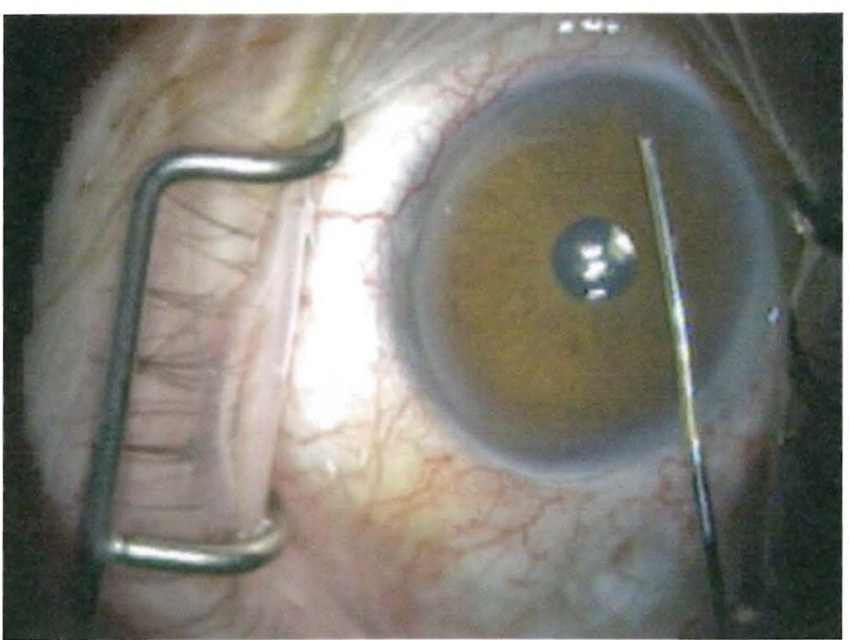

Fig. 8.6 Laser probe is inserted through the paracentesis to cross the anterior chamber (U. Giers)

7. The fiber tip is centered on the pigmented TM and advanced to be in contact with the TM. SC is targeted whenever visible, or surgeons are advised to alternate placement of the fiber to anterior, mid, and posterior TM regions to assure some of the channels will, in fact, enter into SC. The number of pulses which controls the penetration depth is fixed, similar to the penetration depth control in LASIK. Perforation of the inner wall of SC into $\mathrm{SC}$ depends, therefore, on its distance from the fiber tip which can be variable depending on the angle of placement and the amount of pressure on the fiber. The calculation of this distance was determined in numerous preclinical experiments $[28,29]$. Thus, the most common protocol consists of ten probe placement sites on TM, a percentage of which is likely to enter SC.

8. The laser is activated, delivering laser pulses at $20 \mathrm{~Hz}$ per treatment site. Each pulse converts the tissue at the fiber tip into gas. This gas may be seen exiting around the fiber tip during each ablation (Fig. 8.8).

9. As the channel is created, pulse by pulse, an opening is created from the anterior chamber into SC through photoablation of the TM, the juxtacanalicular TM and the inner wall of SC. As soon as the outflow obstruction at the fiber tip is removed as SC is entered, the pathway for the gas, previously retrograde around the fiber, becomes anterograde, entering SC. In theory, this product-ofablation gas is then assumed to dilate SC, pushing the outer wall away and concurrently dilating the adjacent surrounding collector channels. This concept of the product-of-ablation gas dilation of SC and collector channels is termed "pneumatic canaloplasty." Once adequate imaging devices are developed to visualize this process in real time, this theory can be validated.

10. The probe tip is then repositioned such that additional channels are created.
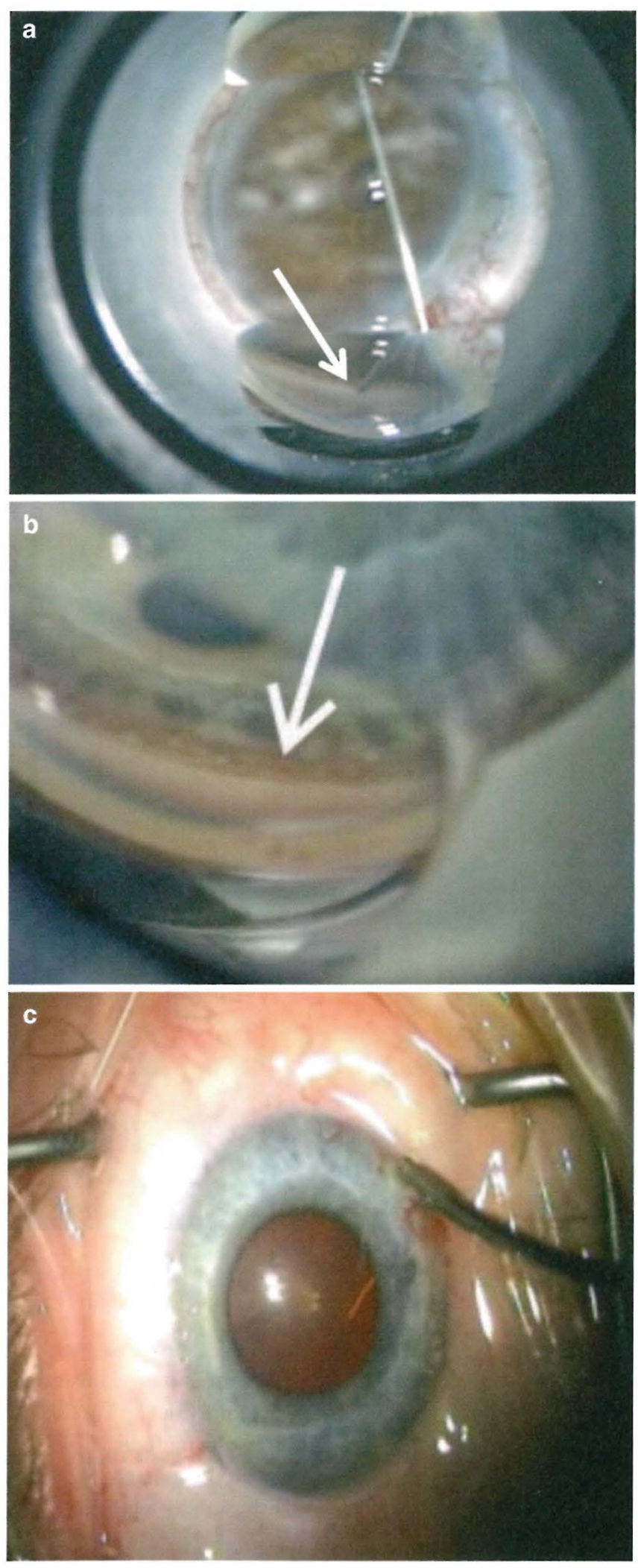

Fig. 8.7 (a) Gonioscopil view of ELT probe in contact with TM (white arrow). (b) Blood in Schlemm's canal (arrow) verifies appropriate targeting and depth, gonioscopic view (c) Induced blood reflux verifies success and enables documentation of number of successful channels into Schlemm's canal (U. Giers) 


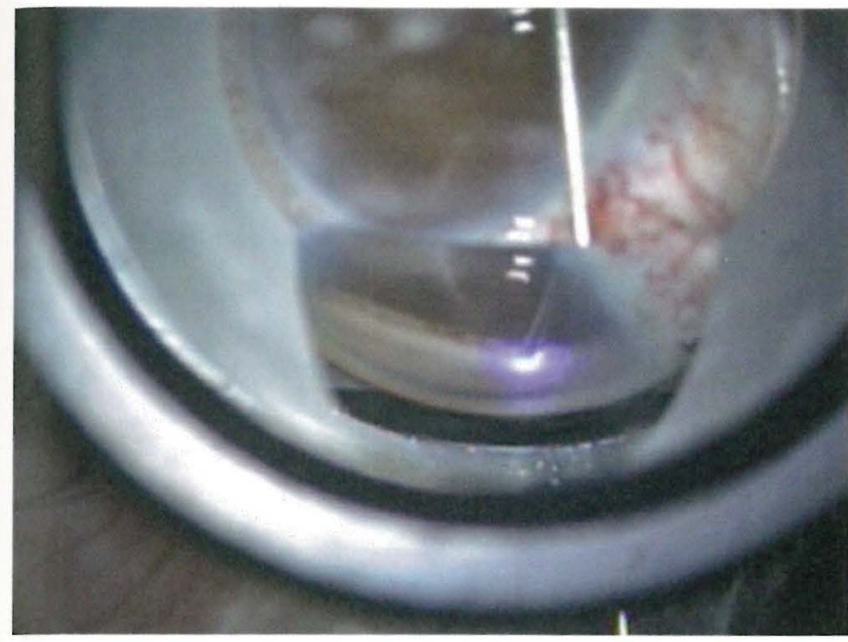

Fig. 8.8 In this series of chanels created tissue fluorescence is visible during a UV laser pulses creating the next channel. (U. Giers)

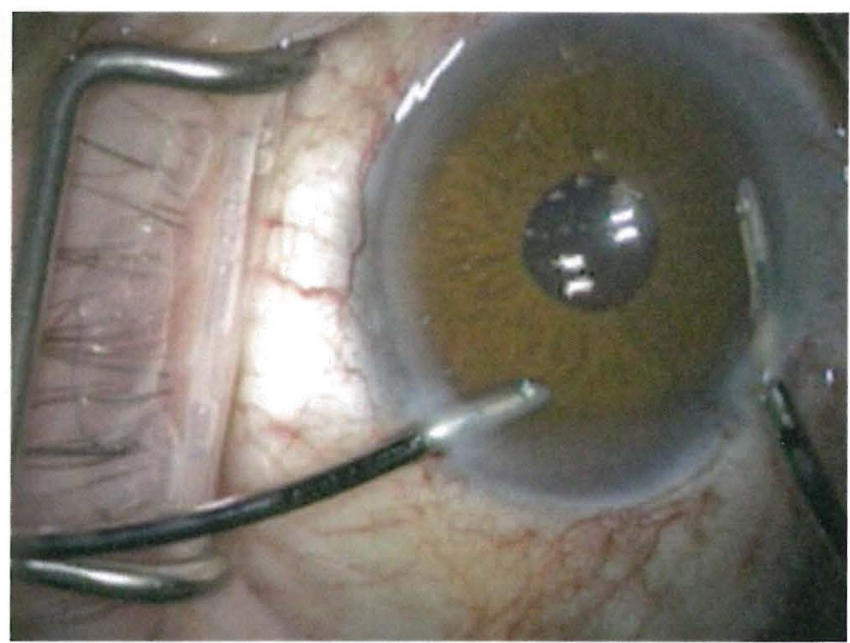

Fig. 8.9 Viscoelastic is exchanged for BSS (U. Giers)

11. The probe is removed from the anterior chamber.

12. The viscoelastic agent is exchanged for BSS with irrigation/aspiration, coaxial or bimanual (Fig. 8.9).

13. The anterior chamber can be monitored for the number of and location of patent trabeculostomy sites by blood reflux from SC during a period of iatrogenic temporary hypotony during the viscoelastic agent/fluid exchange.

Postoperative topical ophthalmic medication regimens are individualized by surgeon, most include:

1. A combination of steroid and antibiotic eye drops or ointment is administered immediately at the end of the procedure.

2. The operated eye is shielded or patched, and the patient is released once stable, similar to after phacoemulsification.

3. The operated eye is usually treated with a topical fixed combination of steroid (dexamethasone $0.1 \%$ ) and antibiotic eye drops qid for 1 week and then tapered over 3 weeks.

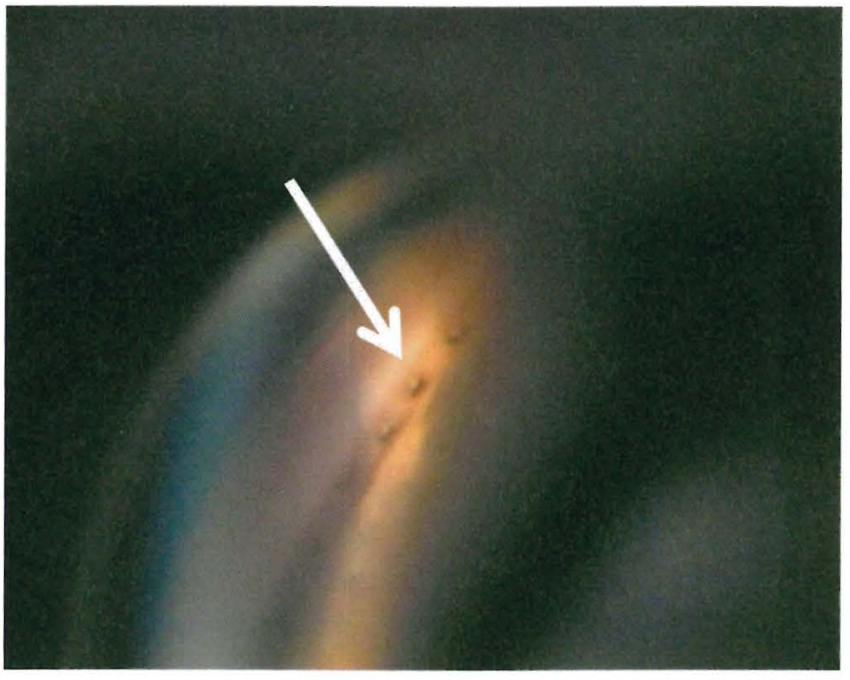

Fig. 8.10 Patent trabeculostomy channels into SC (arrow) documented 2.5 years after ELT (U. Giers)

4. In the rare case of an anterior chamber inflammatory reaction, mydriatic eye drops may be added and the topical steroid can be intensified by surgeon's preference.

Most surgeons discontinue all antiglaucoma medications after the procedure. However, preoperative antiglaucoma medication may be continued and later reduced dependent on the postoperative IOP. When medications are reduced, IOP should be monitored carefully on a regular basis.

\section{After ELT}

Ideally, the patients are monitored postoperatively with gonioscopy in addition to IOP and the findings documented as to the number of sites noted patent. In the cases that have been followed in this manner, channels are documented to remain patent years after the ELT procedure, and in some of these patients, goniolens "pumping" can induce blood to appear at the channels, further confirming the patency of these channels into SC (Figs. 8.10 and 8.11).

\section{ELT Enables Pneumatic Canaloplasty}

Another potential benefit of ELT is that it enables pneumatic canaloplasty. Both endoscopic and gonioscopic views of ELT have revealed gas bubble formation in the tissue and in the anterior chamber as a result of photoablation of the TM tissue. When the ablation penetrates the outflow obstruction, gas is able to enter SC through the newly formed openings in the TM. The pressure of this gas has been proposed to localized dilate SC and collector channels to improve aqueous outflow, thereby lowering IOP. Observing gas bubbles exiting the adjacent openings confirms continuity of flow from SC. 


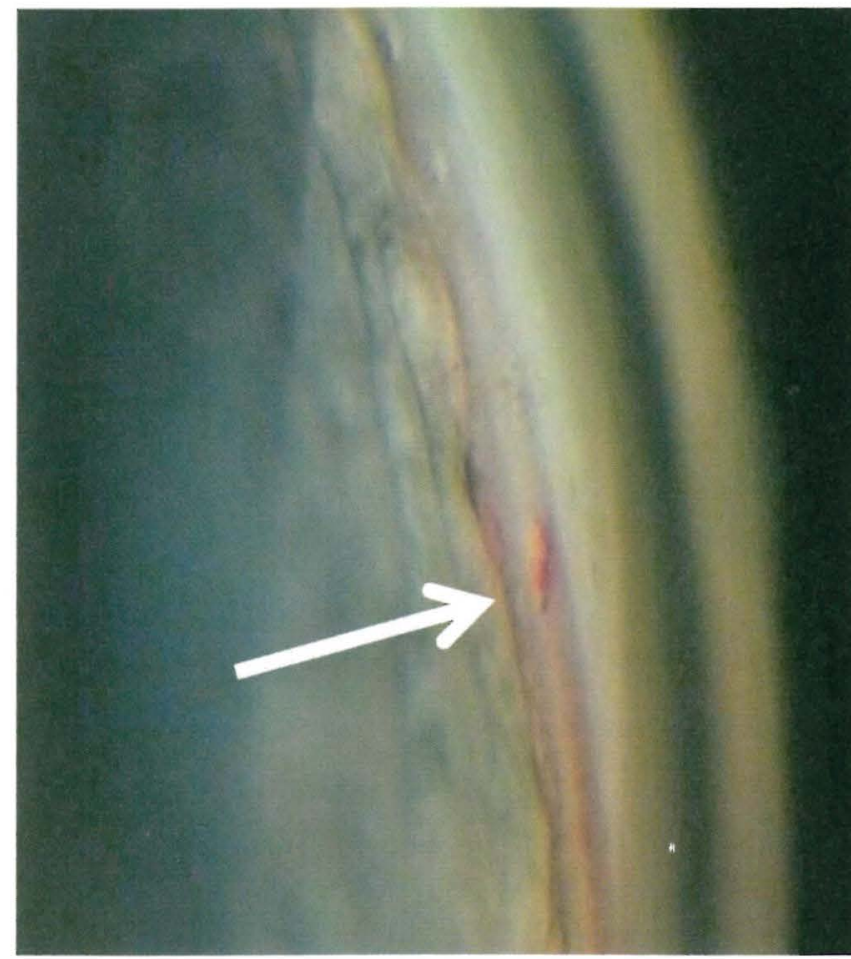

Fig. 8.11 Blood Reflux at the patent opening of a laser channel (white arrow) from SC induced by "pumping" the goniolens 3 years after ELT (U. Giers)

This hypothesis has yet to be confirmed via real-time imaging or histologic studies. Real-time imaging and postmortem histologic studies - in addition to histologic studies after ELT to evaluate the long-term changes which occur subsequent to this procedure - will enable better understanding of the effects and effectiveness of the current procedure and enable suggestions for modifications to potentially further improve the outcomes (Fig. 8.12).

\section{ELT as a MIGS Procedure}

To date the study with the largest sample size was published in 2006 by Pache et al. [30]; included were 135 eyes with openangle glaucoma or ocular hypertension after ELT alone or ELT combined with phacoemulsification. The follow-up was 1 year. Separately two subgroups (subgroup 1 with IOP $>22 \mathrm{mmHg}$ at baseline and subgroup 2 with IOP $\leq 21 \mathrm{mmHg}$ at baseline) were analyzed. Success (IOP $\leq 21 \mathrm{mmHg}, 20 \%$ reduction from baseline, antiglaucoma medications (AGM) $\leq$ baseline, and no subsequent IOP-lowering surgery) was for ELT alone in SG1 $57 \%$ (baseline IOP: $27.9 \pm 3.9 \mathrm{mmHg}$; IOP at 1 year f/u: $19.3 \pm 5.5 \mathrm{mmHg}$ ) and in SG2 $41 \%$ (baseline IOP: $20.2 \pm 1.1 \mathrm{mmHg}$; IOP at 1 year f/u: $17.6 \pm 3.3 \mathrm{mmHg}$ ) and for ELT combined with phacoemulsification, in SG1 $91 \%$ (baseline IOP: $26.4 \pm 2.8 \mathrm{mmHg}$; IOP at 1 year f/u:
$16.7 \pm 2.8 \mathrm{mmHg}$ ) and in SG2 $52 \%$ (baseline IOP: $19.6 \pm 1.1 \mathrm{mmHg}$; IOP at 1 year f/u: $16.3 \pm 2.2 \mathrm{mmHg}$ ). Hence, IOP reduction by ELT appears to be effective in both groups, but much more effective in eyes with higher baseline IOP. This finding has been confirmed by a later study [31].

Wilmsmeyer et al. investigated the outcome after ELT alone (70 eyes) versus ELT combined with phacoemulsification (60 eyes) after a follow-up of 2 years in patients with openangle glaucoma or ocular hypertension [32]. They found a higher reduction of IOP after the combined procedure (IOP reduced from $24.1 \pm 0.7 \mathrm{mmHg}$ to $16.8 \pm 1.0 \mathrm{mmHg}$ at 2 years after ELT alone vs. reduced from $22.4 \pm 0.6 \mathrm{mmHg}$ to $12.6 \pm 1.5$ at 2 years after combined ELT. AGM use did not significantly change).

Babighian et al. found comparable results in an ELT study with 2 years follow-up of 21 eyes with open-angle glaucoma [33]. Success (IOP decrease 20\% with no additional medication, or IOP-lowering surgery) rates were $54 \%$ and IOP was reduced from $24.8 \pm 2.0 \mathrm{mmHg}$ at baseline by $7.9 \pm 0.1 \mathrm{mmHg}$ at 2 years.

Töteberg-Harms et al. were the first to show simultaneous IOP reduction and reduction of antiglaucoma medication after combined ELT (IOP changed from $25.33 \pm 2.85$ at baseline to $16.54 \pm 4.95$ at 1 year, while medications were reduced from $2.25 \pm 1.26$ at baseline to $1.46 \pm 1.38$ at 1 year) [34]. Complete success (IOP $<21 \mathrm{mmHg}$, IOP reduction $\geq 20 \%$, without antiglaucoma medications, and no subsequent IOP-lowering surgery) in their study population was $21.4 \%$ and qualified success (same as complete success but additional antiglaucoma medications were not excluded) $64.3 \%$ at 1 year.

Berlin et al. investigated 46 eyes after combined ELT with a follow-up of 5 years [35]. They showed that IOP was lowered (from $25.5 \pm 6.3$ at baseline to $15.9 \pm 3.0$ at 5 years) and antiglaucoma medications were reduced (from $1.93 \pm 0.87$ at baseline to $0.93 \pm 1.12$ at 5 years) and that this effect remained stable over the entire follow-up of 5 years.

In conclusion, currently published data demonstrates that ELT can reduce IOP and lower antiglaucoma medication simultaneously for up to 5 years. The combined procedure, ELT plus phacoemulsification, appears to be more effective than ELT alone, and the amount of IOP lowering seems to be dependent on baseline IOP and is more effective in eyes with higher baseline IOP.

ELT has shown favorable outcomes in comparison to other outflow procedures (Fig. 8.13). Although ELT, as a laser treatment for glaucoma, is likely to be considered with other glaucoma laser treatments, it is not at all comparable in laser/tissue interaction effects. When compared to office laser treatments, patients undergoing SLT achieved up to a $27 \%$ postoperative decrease in IOP but a negligible decrease in medications. Patients undergoing ALT had similar results, with a maximum of $23.5 \%$ postoperative decrease in IOP and negligible decreases in medications. 

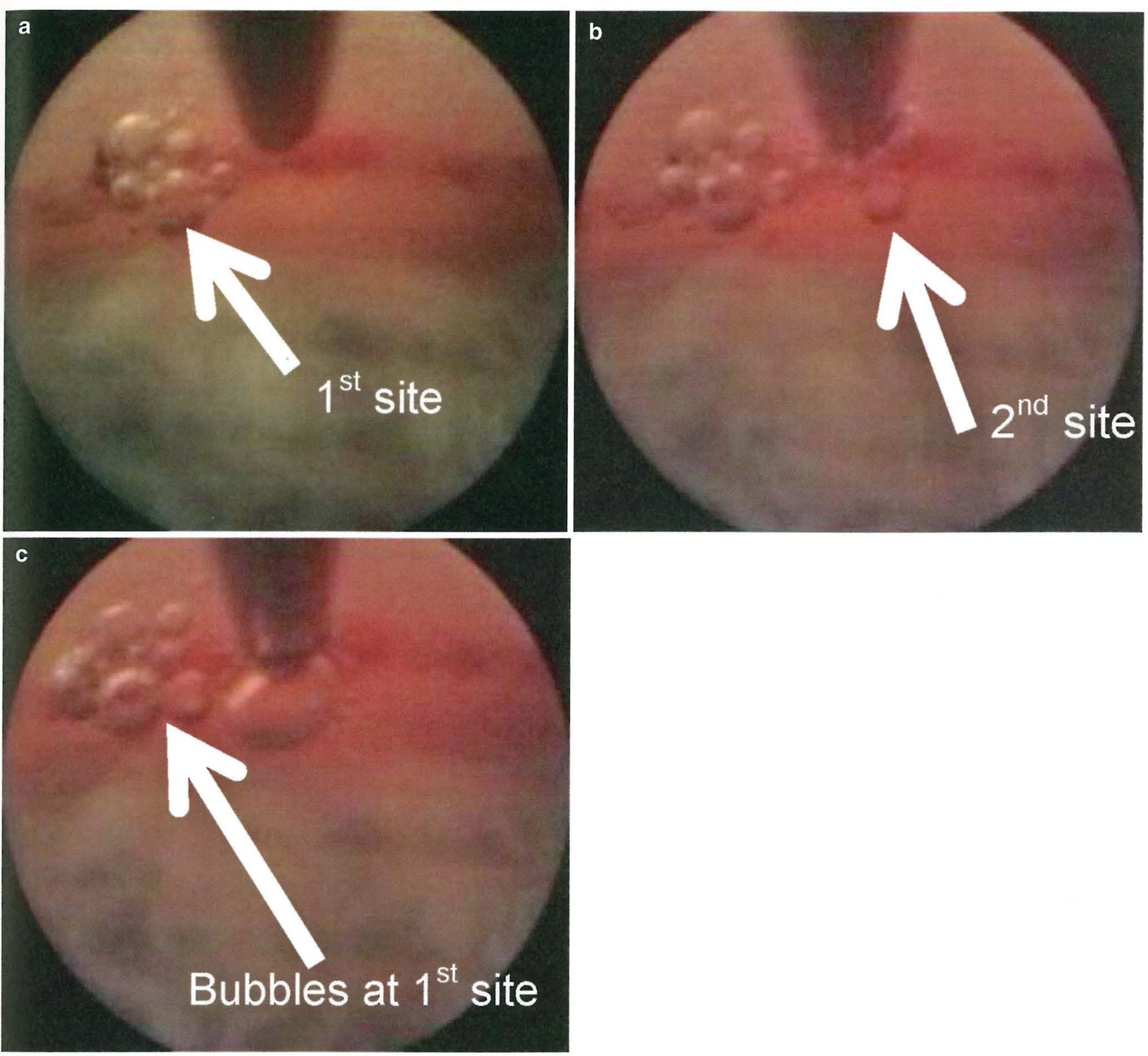

Fig. 8.12 Photos of the ELT procedure demonstrating pneumatic canaloplasty: (a) Coaxial endoscopic view. (b) As the second channel is created into SC, (c) bubble expansion is observed at the first ELT site confirming channel patency into SC at both sites (J. Funk, Zurich, Switzerland)

ELT as an invasive surgical procedure has also shown favorable outcomes when compared to studies of other MIGS procedures. Patients undergoing clear cornea phacoemulsification followed by ab interno gonioscopically guided implantation of one iStent ${ }^{\circledR}$ achieved an IOP decrease of $21.4 \%$ and a medication decrease of $75 \% 1$ year postoperative. Patients undergoing phacoemulsification cataract extraction combined with Trabectome achieved IOP decreases of up to $31.1 \%$ and medication decreases of $41.7 \%$.

Of most relevance is the finding that ELT has shown comparable long-term IOP-lowering results (decrease of $38.6 \%$ after 5 years) to significantly more invasive and risk inherent traditional OR surgeries, trabeculectomy and tube shunt procedures. Though trabeculectomy has demonstrated IOP decreases of $57.1 \%$ and medication decreases of up to $90 \%$, when comparing the data obtained in the Collaborative Initial Glaucoma Treatment Study (CIGTS) on trabeculectomy patients, the 5-year postoperative ELT intraocular pressure measurements at all time points averaged $1 \mathrm{~mm}$ higher than those in the 300 patients who underwent trabeculectomy documented in CIGTS. In addition, the intraoperative complication rate of trabeculectomy was $12 \%$, and the 1 month postoperative complication rate was $50 \%$ versus ELT intraoperative and postoperative complication rates of $0 \%$ $[36,37]$. 


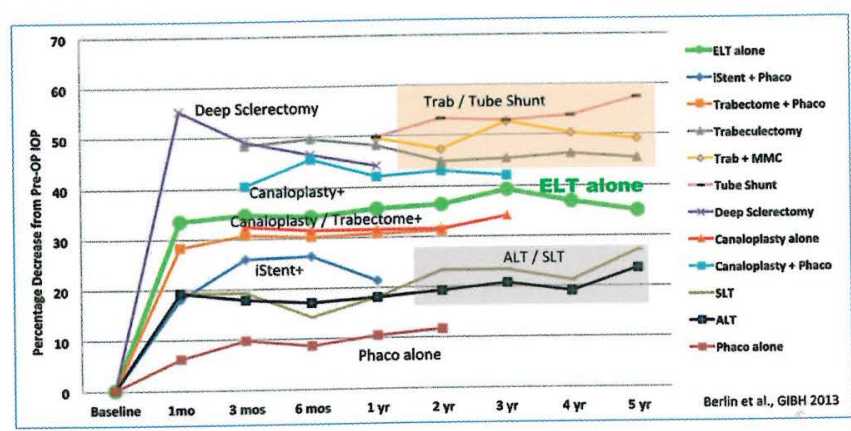

Fig. 8.13 Comparison of various outflow procedures based on the results of exemplary studies. ${ }^{35-44}$

\section{Limitations and Next Generation}

Feedback from leading surgeons who have performed over 2,000 ELT procedures have identified several limitations of the current ELT technique. The next generation of devices, called Guided ELT, is currently under development and is aimed to address the feedback from reporting surgeons. New sensors are being produced to better enable the surgeon to locate SC and TM and to automate the control of the laser, whereas currently tactile contact under observation provides surgeon feedback as to the amount of pressure applied to the meshwork. Furthermore, additional system controls assist in guiding the surgeon as to the depth of tissue removal and number of laser pulses required to penetrate the inner wall of SC with automated detectors and with imaging, especially real-time imaging. The progression of spectral-domain optical coherence tomography development in recent years shows promising abilities to meet these needs.

\section{Conclusion}

ELT is a safe and effective method to reduce both IOP and medication use with minimal complications in most patients with open-angle glaucoma. It is less invasive relative to the methodologies currently being practiced and thus reduces many postoperative issues, including patient discomfort, number of postoperative visits required to assure adequacy and stability, and especially the long-term risks of filtering procedures. By essentially eliminating the damaging and healing response inducing thermal effects and tissue trauma seen with other laser and device procedures, ELT enables the lowering of IOP on a long-term basis. Furthermore, due to the minimal tissue trauma associated with UV tissue photoablation, only a few, small channels into SC have proven adequate to control the IOP. Unlike trabeculectomy, ELT preserves the integrity of the meshwork and SC, which restores the natural outflow without the creation of blebs or invasive foreign body implants. ELT is an important adjunct to cataract surgery, allowing physicians to address two pathologies in one surgical intervention without cutting the conjunctiva.
ELT has been approved for use in the European Union and Switzerland since 1998. Thousands of ELT procedures have been successful in lowering and maintaining lower IOP for years (Fig. 8.13). Currently, clinical studies are pending in both Canada and the United States. We look forward to a better future for our glaucoma patients and especially for their children.

\section{References}

1. Saheb H, Ahmed II. Micro-invasive glaucoma surgery: current perspectives and future directions. Curr Opin Ophthalmol. 2012;23(2):96-104. doi:10.1097/ICU.0b013e32834ff1e7. Review. PubMed PMID: 22249233.

2. Krasnov MM. Laseropuncture of anterior chamber angle in glaucoma. Am J Ophthalmol. 1973;75:674.

3. Hager H. Besondere mikrochirurgische Eingriffe. 2. Teil. Erste Erfahrungen mit dem Argon-Laser-Geraet 800. Klin Monatsbl Augenheilkd. 1973;162:437-50.

4. Van der Zypen E, Fankhauser F, Bebie H, Marshall J. Changes in the ultrastructure of the iris after irradiation with intense light. A study of long-term effects after irradiation with argon ion. Nd:YAG and Q-switched ruby laser. Adv Ophthalmol. 1979;39:59-180.

5. Beckman H, Kinoshita A, Rota AN, et al. Transscleral ruby laser irradiation of the ciliary body in the treatment of intractable glaucoma. Trans Am Acad Ophthalmol Otolaryngol. 1973;46:423.

6. Beckman H, Sugar HS. Neodymium laser cyclocoagulation. Arch Ophthalmol. 1973;90:27.

7. Wise JB. Long-term control of adult open angle glaucoma by argon laser treatment. Ophthalmology. 1981;8:197.

8. Glaucoma Laser Trial Research Group. The Glaucoma Laser Trial (GLT) and glaucoma laser trials follow-up study. 7. Results. Am J Ophthalmol. 1995;120:718.

9. Odberg T, Sandvik L. The medium- and long-term efficacy of primary argon laser trabeculoplasty in avoiding topical medication in open-angle glaucoma. Acta Ophthalmol Scand. 1999;77:176-81.

10. Heijl A, et al. Reduction of IOP and glaucoma progression: results from the early manifest glaucoma trial. Arch Ophthalmol. 2002; 120:1268.

11. Shingleton BJ, et al. Long-term efficacy of argon laser trabeculoplasty: a 10-year follow-up study. Ophthalmology. 1998;100:1324.

12. Damji K, et al. Selective laser trabeculoplasty vs. argon laser trabeculoplasty: a prospective clinical trial. Br J Ophthalmol. 1995; 83:718.

13. Latina MA, Gulati V. Selective laser trabeculoplasty: stimulating the meshwork to mend its ways. Int Ophthalmol Clin. 2004;44:93.

14. Jacobi PC, Dietlein TS, Krieglstein GK. Prospective study of ab externo erbium:YAG laser sclerostomy in humans. Am J Ophthalmol. 1997; 123:478.

15. Iwach AG, et al. Update on the subconjunctival THC:YAG (holmium) laser sclerostomy ab externo clinical trial: a 4-year report. Ophthalmic Surg Lasers. 1996;27:823.

16. Latina MA, Park C. Selective targeting of trabecular meshwork cells: in vitro studies of pulsed and CW interactions. Exp Eye Res. 1995;60:359.

17. Maguen E, Martinez M, Grundfest W, Papaioannou T, Berlin M. Excimer laser ablation of the human lens at $308 \mathrm{~nm}$ with a fiber delivery system. In: XXVI world congress of the International College of Surgeons, Milan, 3-9 July 1988.

18. Tripathy RC. Ultrastructure of Schlemm's canal in relation to aqueous outflow. Exp Eye Res. 1968;7:335-41.

19. Holmberg A. Schlemm's canal and the trabecular meshwork. An electron microscopic study of the normal structure in man and monkey (Cercopithecus aethiops). Doc Ophthalmol. 1965;19:339. 
20. Berlin MS, Rajacich G, Duffy M. Excimer laser photoablation in glaucoma filtering surgery. Am J Ophthalmol. 1987;103: 713-714.3.

21. Huang S, Yu M, Feng G, Zhang P, Qiu C. Histopathological study of trabeculum after excimer laser trabeculectomy ab interno. Yan Ke Xue Bao. 2001;17(1):11-5.

22. Vogel M, Lauritzen K. Selective excimer laser ablation of the trabecular meshwork. Clinical results. Ophthalmologe. 1997;94(9): 665-7.

23. Jahn R, et al. Macroscopic and microscopic findings after excimer laser treatment of different tissue. J Clin Laser Med Surg. 1992; 10:413.

24. Neuhann T, Scharrer A, Haefliger E. Excimer laser trabecular ablation $a b$ interno (ELT) in the treatment of chronic open-angle glaucoma, a pilot study. Ophthalmochirugie. 2001;13:3.

25. Laurtizen K, Vogel M. Trabecular meshwork ablation with excimer laser - a new concept of therapy for glaucoma patients. Invest Ophthalmol Vis Sci. 1997;38:826.

26. Walker R, Specht H. Theoretical and physical aspects of excimer laser trabeculotomy (ELT) ab interno with the AIDA laser operating at a wavelength of $308 \mathrm{~nm}$. Biomed Tech (Berl). 2002; 47:106.

27. Kaufmann R, Hibst R. Pulsed Er:YAG and $308 \mathrm{~nm}$ UV excimer laser: an in vitro and in vivo study of skin-ablative effects. Laser Surg Med. 1989;9:132.

28. Berlin MS. Excimer laser applications in glaucoma surgery. Ophthalmol Clin North Am. 1988;1:255.

29. Berlin MS, et al. Excimer laser photoablation in glaucoma filtering surgery. Am J Ophthalmol. 1984;103:713.
30. Pache M, et al. Laser surgery for glaucoma: excimer-laser trabeculotomy. Klin Monbl Augenheilkd. 2006;223(4):303-7.

31. Töteberg-Harms M, Hanson JV, Funk J. Cataract surgery combined with excimer laser trabeculotomy to lower intraocular pressure: effectiveness dependent on preoperative IOP. BMC Ophthalmol. 2013 Jun 24;13:24. doi:10.1186/1471-2415-13-24. PubMed PMID: 23799932; PubMed Central PMCID: PMC3724507.

32. Wilmsmeyer S, et al. Excimer laser trabeculotomy: a new, minimally invasive procedure for patients with glaucoma. Graefes Arch Clin Exp Ophthalmol. 2006;244:670-6.

33. Babighian $S$, et al. Efficacy and safety of ab interno excimer laser trabeculotomy in primary open-angle glaucoma: two years of follow-up. Ophthalmologica. 2006;220:285-90.

34. Töteberg-Harms M, et al. One-year results after combined cataract surgery and excimer laser trabeculotomy for elevated intraocular pressure. Ophthalmologe. 2011;108(8):733-8.

35. Berlin MS, Kleinberg L, Stodtmeister R, Spitz J, Giers U. The IOP lowering efficacy of excimer-laser-trabeculostomy in open angle glaucoma patients remains consistent over 5 years. Poster session presented at: 23rd annual meeting of the American Glaucoma Society, San Francisco, 1-3 Mar 2013.

36. Stodtmeister R, Kleinberg L, Berlin M, Pillunat L, Giers U. Excimer laser trabeculostomy: 5-year follow-up. Abstract submitted for: the 111th congress of the German Association of Ophthalmologists, Berlin, 19-22 Sept 2013.

37. Jampel HD, Musch DC, Gillespie BW, et al., the CIGTS Study Group. Perioperative complications of trabeculectomy in the Collaborative Initial Glaucoma Treatment Study (CIGTS). Am J Ophthalmol. 2005;140:16-22. 